ORIGINAL RESEARCH

\author{
A.A.K. Abdel Razek \\ S. EIKhamary \\ S. Al-Mesfer \\ H.M. AlKatan
}

\section{Correlation of Apparent Diffusion Coefficient at 3T with Prognostic Parameters of Retinoblastoma}

BACKGROUND AND PURPOSE: Pathologic prognostic parameters for retinoblastoma have been defined. Our purpose was to correlate ADC values at 3T with prognostic parameters of retinoblastoma.

\begin{abstract}
MATERIALS AND METHODS: This study included 72 children (30 boys and 42 girls, mean age $19 \pm 2.6$ months) with retinoblastoma. Pretreatment diffusion-weighted MR imaging was performed on a $3 T$ scanner with b factors of 0,500 , and 1000 seconds $/ \mathrm{mm}^{2}$. ADC values were calculated and pathologic specimens were analyzed. ADC values of the tumors were then correlated with prognostic parameters, including degree of histologic differentiation, tumor size, bilaterality, choroidal invasion, and optic nerve extension.

RESULTS: The mean ADC value of retinoblastoma was $0.49 \pm 0.12 \times 10^{-3} \mathrm{~mm}^{2} / \mathrm{s}$. The ADC values of well- and moderately differentiated tumors were significantly different $(P=.007)$ from poorly and undifferentiated retinoblastoma. There was also a significant difference in the ADC value among small, medium, and large tumors $(P=.015)$, as well as between unilateral and bilateral retinoblastoma $(P=$ $.001)$, and this was independent of the degree of differentiation. The ADC value was also significantly lower $(P=.003)$ when optic nerve invasion was present. There was no correlation of ADC value with growth pattern or choroidal invasion ( $P=.640$ and 0.661 , respectively). The ADC value of retinoblastoma was well correlated with the degree of differentiation of the tumor $(r=0.87, P=.007)$ and inversely correlated with the size of the tumor $(r=-0.68, P=.015)$.
\end{abstract}

CONCLUSIONS: ADC correlated with some of the accepted parameters of poor prognosis for retinoblastoma and may serve as a noninvasive prognostic parameter for assessment of newly diagnosed retinoblastoma.

ABBREVIATIONS: CHESS = chemical shift selective suppression; HE = hematoxylin-eosin; PAS = periodic-acid-Schiff
$\mathbf{R}^{\mathrm{c}}$ etinoblastoma, a highly malignant tumor of the primitive neural retina, is the most common intraocular tumor of childhood. It occurs in 1 of 18,000-30,000 live births worldwide. The average age at diagnosis is 18 months, and $80 \%$ of cases occur during the first 3-4 years of life. The most common clinical sign of retinoblastoma is leukokoria (60\%), followed by strabismus (20\%). ${ }^{1-5}$ Identified pathologic and clinical prognostic parameters of retinoblastoma include degree of histologic differentiation, tumor size, laterality, invasion of the choroid, and extension into the optic nerve. ${ }^{6-9}$ If the optic nerve is not invaded, then the mortality is less than $10 \%$, whereas the mortality rate is $44 \%$ in postlaminar optic nerve invasion. ${ }^{10}$ Bilateral retinoblastomas are aggressive tumors that are commonly associated with metastatic disease and second malignancies. ${ }^{6,7}$ Imaging parameters with prognostic value have also been identified; for example, the degree of contrast enhancement of the anterior eye segment at MR imaging correlates with iris neovascularization, and this is associated with an increased risk of tumor recurrence and metastatic disease. ${ }^{11-13}$

Diffusion-weighted MR imaging is a promising tool in the

Received July 14, 2011; accepted after revision August 22.

From the Diagnostic Radiology Department (A.A.K.A.R.), Mansoura Faculty of Medicine, Mansoura, Egypt; and Radiology Department (S.E.), Pediatric Ophthalmology (S.A.-M.), and Pathology and Laboratory Medicine (H.M.A.), King Khaled Eye Specialist Hospital, Riyadh, Saudi Arabia.

Please address correspondence to Ahmed Abdel Khalek Abdel Razek, Diagnostic Radiology Department, Mansoura Faculty of Medicine, Mansoura, Egypt, 13551; e-mail: arazek@mans.edu.eg

http://dx.doi.org/10.3174/ajnr.A2892 evaluation of head and neck malignancy ${ }^{14,15}$ and orbital disease. Diffusion-weighted MR imaging has been used in evaluation of orbital tumors of adults ${ }^{16,17}$ and children, ${ }^{18}$ ocular adnexal lymphomas, ${ }^{19}$ endophtalmitis, ${ }^{20}$ and optic nerve lesions. ${ }^{21,22}$ Several studies have correlated the apparent diffusion coefficient value with prognostic parameters for breast and lung cancer. ${ }^{23-25}$ The aim of this work was to correlate the ADC value with prognostic parameters of retinoblastoma.

\section{Materials and Methods}

A retrospective study approved by our institutional review board was conducted upon consecutive patients with retinoblastoma who underwent diffusion-weighted MR imaging between January 2007 and November 2010. The inclusion criteria were 1) previously untreated patients with unilateral or bilateral retinoblastoma who underwent pre- and postcontrast and diffusion MR imaging of the orbit, and 2) subsequent enucleation of the affected eye in unilateral retinoblastoma and of 1 eye in bilateral retinoblastoma. Seventy-nine patients met these inclusion criteria, but 7 were subsequently excluded from the study: 3 patients had poor quality images due to susceptibility artifacts at MR imaging, and the quality of 4 pathologic specimens hampered proper analysis. Seventy-two patients, ranging in age from 1 month to 29 months (mean $=19 \pm 2.6$ months), were therefore included in this study. Patients with unilateral retinoblastoma ( $n=$ 49 ) underwent unilateral enucleation, and patients with bilateral retinoblastoma $(n=23)$ underwent unilateral enucleation for treatment of the more advanced lesion.

MR imaging was performed at 3T (Magnetom Allegra; Siemens, Erlangen, Germany), with use of a dedicated head coil. The gradient strength was $40 \mathrm{mT} / \mathrm{m}$ and slew rate was $400 \mathrm{~T} / \mathrm{m} / \mathrm{s}$. All children were 

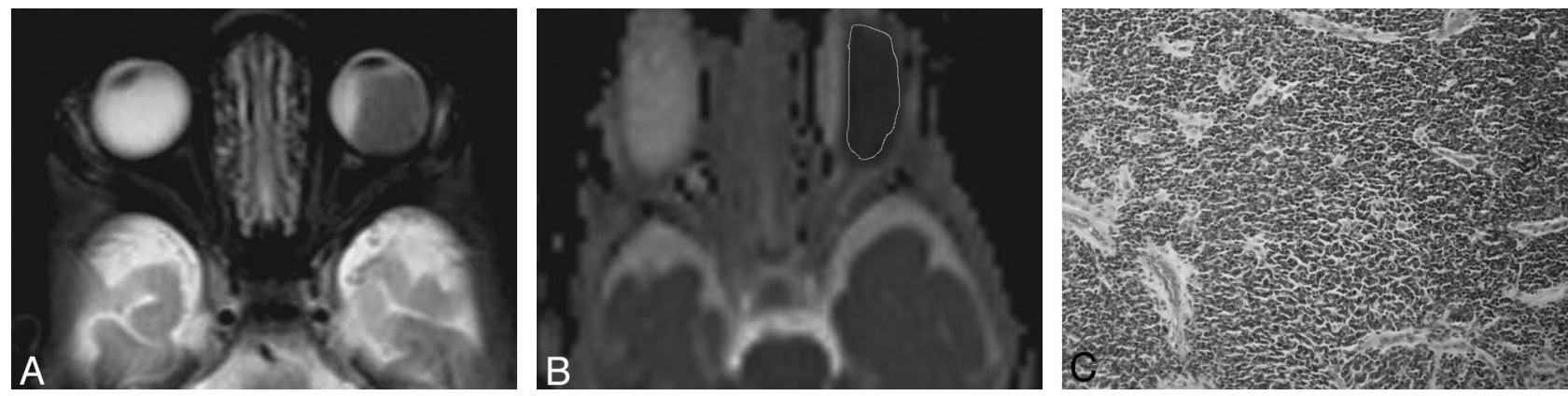

Fig 1. Unilateral large poorly differentiated retinoblastoma. $A$, Axial contrast T2-weighted image shows large tumor in the left globe. $B$, ADC map shows ROI localization of the tumor. There is restricted diffusion with low $A D C$ value $\left(0.44 \times 10^{-3} \mathrm{~mm}^{2} / \mathrm{s}\right)$ of the tumor. $C$, Pathologic specimen shows poorly differentiated retinoblastoma with proliferating small round to polygonal cells that show hyperchromatic nuclei and minimal cytoplasm $\times 100 \mathrm{H} \& \mathrm{E}$.

sedated with an oral mixture of $1 \mathrm{mg} / \mathrm{kg}$ of promethazine hydrochloride (Centrafarm Services, Etten-Leur, Netherlands). Axial and coronal fat suppressed T2-weighted MR images were obtained with TR/TE of 4000-4600/19-96 ms, FOV of $20 \times 22 \mathrm{~cm}$, section thickness of 3 $\mathrm{mm}$, intersection gap of $1 \mathrm{~mm}$, and matrix of $320 \times 260$. Diffusionweighted MR images were obtained using a multisection spin-echo echo-planar imaging sequence. Imaging parameters were TR/TE of $3200 / 80 \mathrm{~ms}$, FOV of $20 \times 22 \mathrm{~cm}$, section thickness of $3 \mathrm{~mm}$, intersection gap of $1 \mathrm{~mm}$, number of excitation of 6 , matrix of $128 \times 128$, EPI factor of 128, and radio-frequency pulse bandwidth of $1200 \mathrm{~Hz} /$ pixel. We used the automatic multiangle-projection shim and CHESS technique to reduce the artifacts in diffusion-weighted MR images. Diffusion probing gradients were applied in the 3 orthogonal directions $(x, y$, and $z)$ with the same strength. Diffusion-weighted MR images were acquired with $b$ factors of 0,500 , and $1000 \mathrm{~mm}^{2} / \mathrm{s}$, and ADC maps were automatically generated on the operating console. The data acquisition time for the diffusion-weighted images was 90-120 seconds for all 3 values together. Contrast-enhanced fat-suppressed T1-weighted images (TR/TE of 400-575/13-15 ms) in axial, coronal, and sagittal oblique (along the course of the optic nerves) planes were then obtained following intravenous injection of $0.1 \mathrm{~mL} / \mathrm{kg}$ of body weight gadopentate dimeglumine (Omniscan; Schering, Berlin, Germany).

Quantitative analysis of the ADC map was performed by 1 radiologist (E.S.) with 15 years' experience in MR imaging. An ROI was manually traced on the ADC map using an electronic cursor (Fig 1). The ROI was smaller in size than actual tumor size to exclude adjacent normal tissue and vitreous. In the case of bilateral tumors, the ADC value of the enucleated eye was used for analysis. In the case of necrotic tumors with heterogeneous contrast enhancement, 3 ROIs were placed within the solid part of the tumor to avoid bias from necrotic elements, which give falsely elevated ADC values; the final $\mathrm{ADC}$ value of the tumor was the average of the ADC of these 3 ROIs. The longest diameter of the tumor at contrast-enhanced T1-weighted image was calculated. The tumor diameters were classified as $<10$ $\mathrm{mm}$, between $10-15 \mathrm{~mm}$, and $>15 \mathrm{~mm}$.

One pathologist (H.M.A.) with 12 years' experience in ocular pathology examined all histopathological sections. The data from the whole-eye sections cut parallel to the optic nerve, with a thickness of 6- $\mu \mathrm{m}$ and stained with HE and PAS reaction were analyzed. All histopathologic slides were reviewed for growth pattern of the tumor, degree of tumor differentiation, and extent of choroidal and optic nerve involvement. Regarding growth pattern, tumor growth is endophytic when tumors grow within the vitreous cavity, exophytic when the growth is subretinal, or can be combined. Choroidal inva- sion is diagnosed by the presence of a tumor between the retina and sclera. Tumor extension into the optic nerve is categorized with reference to the lamina cribrosa as pre- or postlaminar invasion. Tumors were also graded as well differentiated, moderately differentiated, poorly differentiated, or undifferentiated. The degree of tumor differentiation was based on the percentage of differentiated areas (FlexnerWintersteiner rosettes and fleurettes) in relation to the total tumor area. $^{26}$

Statistical analysis of data was performed using SPSS version 10 (SPSS, Chicago, Illinois). All data were revealed to be parametric with a normal distribution by the Kolmogorov-Smirnov Test. The mean and standard deviation of the ADC value for each prognostic factor (tumor size, bilaterality, growth pattern, choroidal invasion, optic nerve invasion, and degree of differentiation) was calculated. A Student $t$-test was used to compare 2 groups, and the ANOVA test was used to compare more than 2 groups. The $P$ value was considered significant if $\leq .05$ at the confidence interval of $95 \%$. Fisher exact correlation test was used to determine the strength of relationship between the ADC value and tumor size and degree of differentiation.

\section{Results}

All retinoblastomas were hyperintense on diffusion-weighted images, with $b=1000 \mathrm{~mm}^{2} / \mathrm{s}$. The mean ADC value of the retinoblastomas was $0.49 \pm 0.12 \times 10^{-3} \mathrm{~mm}^{2} / \mathrm{s}(0.20-0.86 \times$ $\left.10^{-3} \mathrm{~mm}^{2} / \mathrm{s}\right)$. The Table presents the minimum, maximum, mean, and $\mathrm{SD}$ of $\mathrm{ADC}$ values of the retinoblastomas in relation to previously defined prognostic parameters. ${ }^{6}$

The well- and moderately differentiated retinoblastomas showed Flexner-Wintersteiner rosettes and fleurettes. The poorly differentiated and undifferentiated tumors revealed high nuclear cytoplasmic ratios and hyperchromatic nuclei. The mean ADC value of well-differentiated retinoblastomas $\left(0.54 \pm 0.20 \times 10^{-3} \mathrm{~mm}^{2} / \mathrm{s}\right.$; Fig 2$)$ and moderately differentiated retinoblatomas $\left(0.51 \pm 0.07 \times 10^{-3} \mathrm{~mm}^{2} / \mathrm{s}\right)$ was significantly different $(P=.007)$ from poorly differentiated $(0.44 \pm$ $\left.0.07 \times 10^{-3} \mathrm{~mm}^{2} / \mathrm{s}\right)$ and undifferentiated retinoblastomas $\left(0.41 \pm 0.01 \times 10^{-3} \mathrm{~mm}^{2} / \mathrm{s}\right.$; Figs 1 and 3$)$. The ADC value of the tumors was well correlated with the degree of tumor differentiation $(r=0.87, P=.007)$.

The ADC value of small tumors ( $\leq 10 \mathrm{~mm}, n=11)$ was $0.55 \pm 0.09 \times 10^{-3} \mathrm{~mm}^{2} / \mathrm{s}$ and of medium-sized tumors $(>10$ $\mathrm{mm}$ to $15 \mathrm{~mm}, n=33$ ) was $0.48 \pm 0.09 \times 10^{-3} \mathrm{~mm}^{2} / \mathrm{s}$ (Fig 2). Tumors more than $15 \mathrm{~mm}(n=28)$ had an ADC value of $0.38 \pm 0.11 \times 10^{-3} \mathrm{~mm}^{2} / \mathrm{s}$. There was a significant difference in the ADC value among small, medium-sized, and large tu- 


\begin{tabular}{|c|c|c|}
\hline \multicolumn{3}{|c|}{$\begin{array}{l}\text { The minimum, maximum, mean, and standard deviation of ADC } \\
\text { value of retinoblastoma in relation to prognostic parameters }\end{array}$} \\
\hline Prognostic Parameter & ADC Value & $P$ Value \\
\hline \multicolumn{3}{|l|}{ Pathologic grade } \\
\hline Well differentiated $(n=16)$ & $0.54 \pm 0.20(0.41-0.86)$ & $0.007^{*}$ \\
\hline Moderately differentiated $(n=20)$ & $0.51 \pm 0.07(0.33-0.70)$ & \\
\hline Poorly differentiated $(n=7)$ & $0.44 \pm 0.07(0.23-0.57)$ & \\
\hline Undifferentiated $(n=29)$ & $0.41 \pm 0.01(0.20-0.59)$ & \\
\hline \multicolumn{3}{|l|}{ Size } \\
\hline Small (less than $10 \mathrm{~mm} ; n=11$ ) & $0.55 \pm 0.09(0.36-0.72)$ & $0.015^{*}$ \\
\hline Medium (10-15 mm; $n=36)$ & $0.48 \pm 0.08(0.34-0.57)$ & \\
\hline Large (more than $15 \mathrm{~mm} ; n=25$ ) & $0.38 \pm 0.11(0.20-0.86)$ & \\
\hline \multicolumn{3}{|l|}{ Side } \\
\hline Unilateral $(n=49)$ & $0.53 \pm 0.11(0.34-0.86)$ & $0.001^{*}$ \\
\hline Bilateral $(n=23)$ & $0.35 \pm 0.11(0.20-0.51)$ & \\
\hline \multicolumn{3}{|l|}{ Growth pattern } \\
\hline Endophytic $(n=18)$ & $0.49 \pm 0.16(0.23-0.72)$ & 0.640 \\
\hline Exophytic $(n=19)$ & $0.49 \pm 0.11(0.25-0.70)$ & \\
\hline Combined $(n=35)$ & $0.46 \pm 0.15(0.20-0.86)$ & \\
\hline \multicolumn{3}{|l|}{ Choroidal invasion } \\
\hline Choroidal invasion $(n=34)$ & $0.48 \pm 0.13(0.20-0.86)$ & 0.661 \\
\hline No invasion $(n=38)$ & $0.47 \pm 0.15(0.23-0.84)$ & \\
\hline \multicolumn{3}{|l|}{ Optic nerve invasion } \\
\hline Postlaminar $(n=8)$ & $0.38 \pm 0.11(0.20-0.55)$ & $0.003^{*}$ \\
\hline Prelaminar $(n=15)$ & $0.39 \pm 0.23(0.25-0.60)$ & \\
\hline No invasion $(n=49)$ & $0.52 \pm 0.10(0.40-0.86)$ & \\
\hline
\end{tabular}

${ }^{*}$ indicates significant $P$ values.

mors $(P=.015)$, with the ADC value inversely correlated with tumor size $(r=-0.68, P=.015)$.

Retinoblastoma was unilateral in 49 patients and bilateral in 23 patients. Bilateral retinoblastomas were poorly differentiated or undifferentiated in all cases. The ADC value of the retinoblastoma in the enucleated eye in patients with bilateral lesions was $0.35 \pm 0.11 \times 10^{-3} \mathrm{~mm}^{2} / \mathrm{s}$ (Fig 3) and of unilateral retinoblastoma was $0.54 \pm 0.12 \times 10^{-3} \mathrm{~mm}^{2} / \mathrm{s}$. There was significant difference in the ADC value between bilateral and unilateral retinoblastomas $(P=.001)$.

The ADC value of endophytic tumors $(n=18)$ was $0.49 \pm$ $0.16 \times 10^{-3} \mathrm{~mm}^{2} / \mathrm{s}$ and that of exophytic tumors $(n=19)$ was $0.49 \pm 0.11 \times 10^{-3} \mathrm{~mm}^{2} / \mathrm{s}$. The ADC of tumors with combined growth pattern $(n=35)$ was $0.46 \pm 0.15 \times 10^{-3} \mathrm{~mm}^{2} / \mathrm{s}$. There was no significant difference in the ADC value of endophytic, exophytic, and combined forms of retinoblastoma $(P=.640)$. There was also no significant difference in the ADC of retinoblastoma with or without choroidal invasion $(P=$ $.661)$.

Optic nerve invasion was prelaminar in 15 eyes and postlaminar in 8 eyes. The ADC value of retinoblastoma with pathologically proved postlaminar invasion $\left(0.38 \pm 0.11 \times 10^{-3} \mathrm{~mm}^{2} / \mathrm{s}\right.$; Fig 4) was lower than that of pathologically proved prelaminar invasion $\left(0.39 \pm 0.23 \times 10^{-3} \mathrm{~mm}^{2} / \mathrm{s}\right)$, but it did not reach significance. The ADC value of retinoblastoma in patients with optic nerve invasion $\left(0.39 \pm 0.15 \times 10^{-3} \mathrm{~mm}^{2} / \mathrm{s}\right)$ was, however, significantly different $(P=.003)$ from that of patients without optic nerve invasion $\left(0.50 \pm 0.10 \times 10^{-3} \mathrm{~mm}^{2} / \mathrm{s}\right)$.

\section{Discussion}

Retinoblastomas with high-risk features (including a lack of differentiation, large size, bilaterality, choroidal infiltration, or retrolaminar optic nerve invasion) are likely to have a poor prognosis and clinical outcome. ${ }^{6-10} \mathrm{We}$ evaluated the poten- tial of diffusion-weighted MR imaging to characterize retinoblastoma by determining ADC values and correlating these with traditional prognostic parameters.

Distinction between poorly and well-differentiated tumors impacts both prognosis and treatment planning. Well-differentiated tumors have a good prognosis and are radioresistent, whereas poorly differentiated tumors have a worse prognosis and respond to radiation therapy. ${ }^{27,28}$ Well-differentiated tumors contain rosettes or fleurettes: Flexner-Wintersteiner rosettes are composed of cells arranged in a circular fashion around a well-defined empty central zone, while fleurettes are benign-appearing cells that show photoreceptor differentiation with formation of bouquet-like clusters without mitosis. Poorly differentiated tumors are composed of small round cells with a high nuclear to cytoplasmic ratio and hyperchromatic nuclei that are devoid of Flexner-Wintersteiner rosettes and fleurettes. ${ }^{28,29}$ Diffusion-weighted MR imaging is a fast sequence that can be added to routine MR imaging and may provide information about cellularity of the tumor ${ }^{14,15}$ via the $\mathrm{ADC}$ value; the ADC value is correlated with tumor differentiation and this information cannot be obtained with conventional MR imaging. The ADC value of well- or moderately differentiated malignancies has been demonstrated to be significantly different from that of poorly differentiated cancers in many different regions of the body. ${ }^{14,15,23}$ In this study, the ADC value of poorly and undifferentiated retinoblastoma is statistically different $(P=.007)$ from well- and moderately differentiated tumors. Hypercellularity and enlarged nuclei of poorly differentiated retinoblastomas reduce the extracellular matrix and the diffusion space of water protons in the extracellular and intracellular dimensions, with a resultant decrease in the ADC value of the tumor.

Tumor size is a valuable prognostic factor of retinoblastoma, as larger tumors are typically associated with a worse clinical outcome, and the diameter of $15 \mathrm{~mm}$ is commonly used for denoting poor prognosis. ${ }^{6-10}$ In this study, large tumors had lower ADC values than small tumors, but there is overlap in their ADC values. This may be attributed to large tumors commonly having more compact cells with more restricted diffusion, but they may be associated with microscopic areas of necrosis with potentially free diffusion. A previous study reported that there is a significant difference $(P=$ $.001)$ in the ADC value between small and large invasive ductal carcinomas of the breast. ${ }^{24}$ On the other hand, there was no significant difference in the ADC value between $\mathrm{T}$ stages of lung cancer. ${ }^{25}$

Bilateral retinoblastomas are generally heritable and accompanied by germ-line mutation of the $R b 1$ gene, but they may be nonheritable and occur spontaneously. Patients with inherited retinoblastoma present earlier than those with nonheritable tumors. Bilateral retinoblastomas, comprising approximately three-quarters of cases of retinoblastoma, are aggressive tumors that commonly develop metastatic disease. ${ }^{30,31}$ In this work, the ADC value of bilateral tumors is significantly lower than that of unilateral disease. This was attributable to all bilateral retinoblastomas being poorly or undifferentiated tumors at histopathologic examination, with subsequent lower ADC value than unilateral disease.

In this study, there was no significant difference in the ADC 

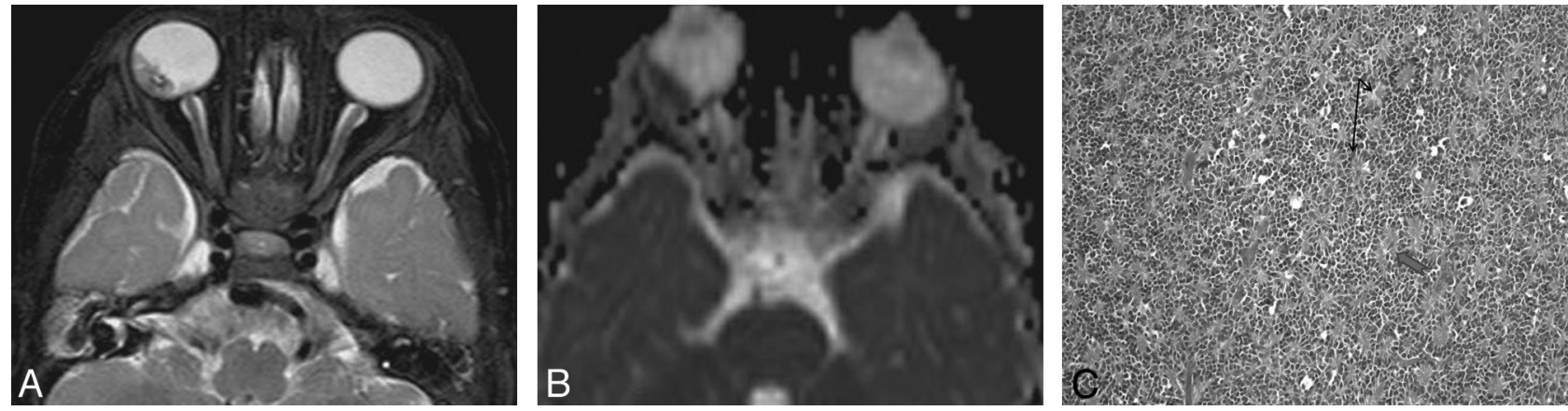

Fig 2. Unilateral medium-sized well-differentiated retinoblastoma. $A$, Axial T2-weighted image shows hypointense mass in the right globe, measuring 10 mm. $B$, ADC map shows restricted diffusion of the tumor with low ADC value $\left(0.47 \times 10^{-3} \mathrm{~mm}^{2} / \mathrm{s}\right)$. C, Pathologic specimen shows Flexner-Wintersteiner rosettes (black arrows) and fleurettes (large blue arrow), which indicate differentiation in retinoblastoma $\times 400$ PAS.
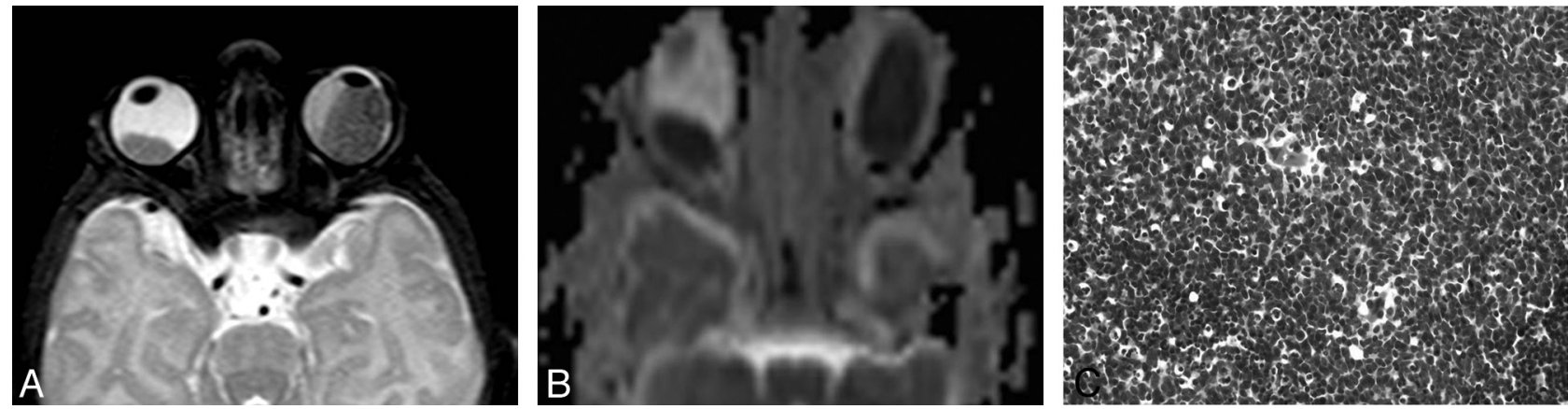

Fig 3. Bilateral retinoblastoma. $A$, Axial T2-weighted image shows bilateral retinoblastomas with large tumor on left side and small tumor on the right side. $B$, ADC map shows restricted diffusion of both lesions. The ADC value of the left-side large tumor is $0.36 \times 10^{-3} \mathrm{~mm}^{2} / \mathrm{s}$ and of right-side small tumor is $0.41 \times 10^{-3} \mathrm{~mm} / \mathrm{s}$. $C$, Pathologic specimen of large left-sided tumor shows poorly differentiated retinoblastoma with proliferating small, round to polygonal cells, which show hyperchromatic nuclei and minimal cytoplasm $\times 400 \mathrm{H} \& \mathrm{E}$.
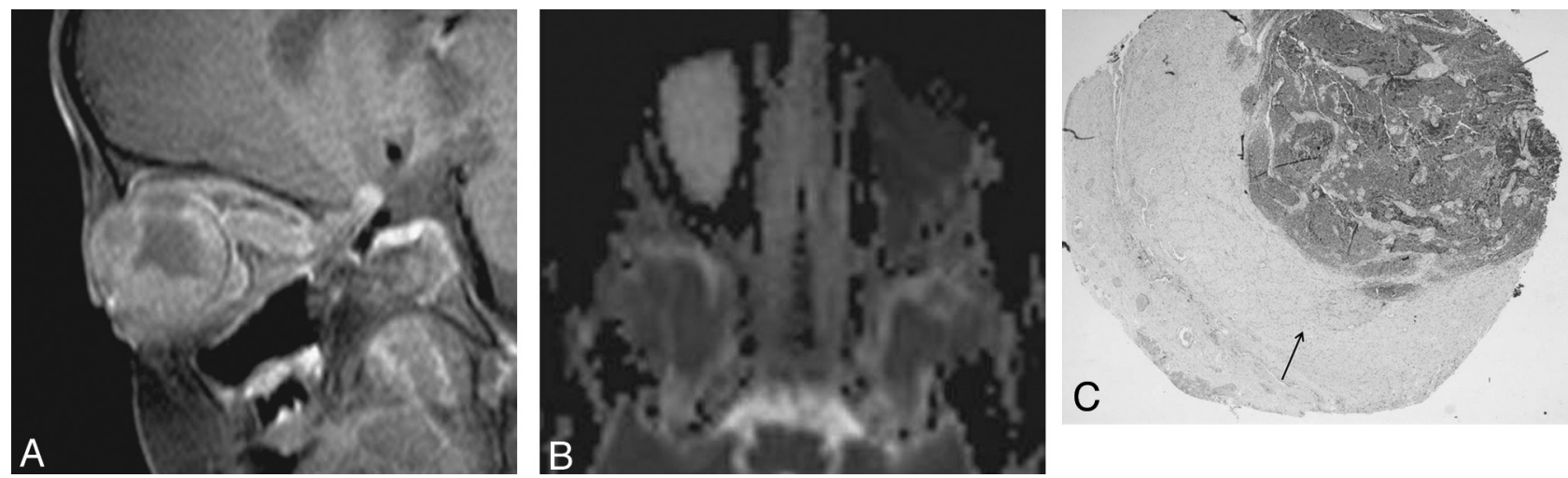

Fig 4. Retinoblastoma with optic nerve invasion. $A$, Sagittal oblique contrast T1-weighted image shows retinoblastoma with extension into the optic nerve. $B$, $A D C$ map shows restricted diffusion with low ADC value of retinoblastoma from the solid part of the tumor $\left(0.33 \times 10^{-3} \mathrm{~mm}^{2} / \mathrm{s}\right)$. Note extension of the tumor into the left optic nerve with restricted diffusion. $C$ Pathologic specimen shows optic nerve resection margin with invasion of retinoblastoma (red arrow) into the substance of the optic nerve (black arrow) $\times 25$ PAS.

values among the different growth patterns of retinoblastoma, which may be attributable to the effect of retinal detachment and associated subretinal fluid. The growth pattern of retinoblastoma can be described according to the retinal spread of the tumor. Endophytic tumors arise from inner layers of the retina and grow into the vitreous. Exophytic tumors start in the outer layers and growth is in the subretinal space. Exophytic growth patterns increase risk of metastasis due to earlier choroidal involvement and a high percentage of patients develop glaucoma. ${ }^{6-10}$

Involvement of the choroid increases the risk of metastatic disease because of its rich vascular network, and choroidal invasion is usually associated with a higher mortality rate.
Choroidal invasion may be seen as a local thickening of the choroid or a discontinuity in normal linear enhancement beneath the retinoblastoma. ${ }^{4,32,33}$ In this study, there is no correlation between the degree of choroidal infiltration at histologic analysis and the ADC value of retinoblastoma. This may be attributable to the ADC value being measured from the entire tumor and not just the part of the tumor that invaded the choroid. In addition, choroidal invasion is generally focal, and hence it may have insignificant effect on the ADC value.

Optic nerve invasion, especially postlaminar, is a marker of tumor aggressiveness and is associated with a significant increase in metastatic disease and mortality rate. ${ }^{10,34}$ Abnormal contrast enhancement and enlargement of the optic nerve are 
used as diagnostic criteria for postlaminar optic invasion. In this work, lower ADC values are observed in tumors with optic nerve invasion. This may be attributed to retinoblastoma, with optic nerve invasion generally being a poorly differentiated or undifferentiated tumor with higher cellularity than a tumor without optic nerve invasion.

In this study, we applied $3 \mathrm{~b}$-values to obtain 1 ADC map using 3-mm sections and short echo times to improve image quality, and this was facilitated by high-field 3T imaging. ${ }^{14-17}$ Recent advances in MR software technology at 3T will likely continue to improve image quality and also promise the potential application of multiple and higher b-values than 1000 $\mathrm{mm}^{2} / \mathrm{s}$, which may increase diffusion sensitivity and allow further characterization of these tumors on the basis of ADC.

There are several limitations of our study. First, we applied diffusion-weighted MR imaging but did not perform diffusion tensor imaging. Further studies with application of diffusion tensor MR imaging with multichannel coils will likely improve the detection of optic nerve invasion and small lesions. Second, we excluded 3 patients due to susceptibility artifacts, and we expect that future studies with application of parallel imaging will increase image contrast and decrease susceptibility artifacts. $^{14-15}$ Third, there is potential bias related to ROI selection as well as the presence or absence of retinal detachment, associated subretinal fluid, and/or tumor necrosis. Application of new postprocessing methods such as nonGaussian (diffusion kurtosis) modeling or K-means clustering algorithms may provide a better characterization of different components of the tumor compared with whole lesion mean ADC value. ${ }^{14,15}$ Fourth, we have not yet correlated ADC values with genetic studies in bilateral retinoblastomas. Finally, we have not performed any correlation with long-term outcome; further studies with long-term follow-up will be needed to correlate the ADC values with survival of the patients.

\section{Conclusions}

The ADC of retinoblastoma correlates with some of the accepted parameters of poor prognosis. Patients with negative prognostic parameters - such as poorly differentiated or undifferentiated tumor, bilaterality, large tumors, and optic nerve invasion-tend to have lower apparent diffusion coefficient. Hence, the ADC can be considered a noninvasive prognostic parameter of retinoblastoma that reflects the degree of tumor differentiation and that may identify highly aggressive tumors.

\section{References}

1. Apushkin MA, Apushkin MA, Shapiro MJ, et al. Retinoblastoma and simulating lesions: role of imaging. Neuroimaging Clin North Am 2005;15:49-67

2. Kadom N, Sze R. Radiological reasoning: leukocoria in a child. AJR Am J Roentgenol 2008;191:S40-44

3. Razek A, Elkhamary S. MR imaging of retinoblastoma. Br J Radiol 2011;84: 755-84

4. Saket RR, Mafee MF. Anterior-segment retinoblastoma mimicking pseudoinflammatory angle-closure glaucoma: review of the literature and the important role of imaging. AJNR Am J Neuroradiol 2009;30:1607-09

5. Chung E, Specht C, Schroeder J. Pediatric orbit tumors and tumorlike lesions: neuroepithelial lesions of the ocular globe and optic nerve. RadioGraphics 2007;27:1159-86

6. Balaguer J, Wilson M, Billups $\mathrm{C}$, et al. Predictive factors of invasion in eyes with retinoblastoma enucleated after eye salvage treatments. Pediatr Blood Cancer 2009;52:351-56

7. Atchaneeyasakul L, Wongsiwaroj C, Uiprasertkul M, et al. Prognostic factors and treatment outcomes of retinoblastoma in pediatric patients: a single-institution study. Jpn J Ophthalmol 2009;53:35-39

8. Gupta R, Vemuganti G, Reddy V, et al. Histopathologic risk factors in retinoblastoma in India. Arch Pathol Lab Med 2009;133:1210-14

9. Finger PT, Harbour J, Karcioglu Z. Risk factors for metastasis in retinoblastoma. Surv Ophthalmol 2002;47:1-16

10. Brisse $H$, Guesmi M, Aerts I, et al. Relevance of CT and MRI in retinoblastoma for the diagnosis of postlaminar invasion with normal-size optic nerve: a retrospective study of 150 patients with histologic comparison. Pediatr Radiol 2007;37:649-56

11. de Graaf P, van der Valk P, Moll A, et al. Contrast-enhancement of the anterior eye segment in patients with retinoblastoma: correlation between clinical, MR imaging, and histopathologic findings. AJNR Am J Neuroradiol 2010;31:237-45

12. Galluzzi P, Cerase A, Hadjistilianou T, et al. Retinoblastoma: abnormal gadolinium enhancement of anterior segment of eyes at MR imaging with clinical and histopathologic correlation. Radiology 2003;228:683-90

13. de Graaf P, Barkhof F, Moll A, et al. Retinoblastoma: MR imaging parameters in detection of tumor extent. Radiology 2005;235:197-207

14. Schafer J, Srinivasan A, Mukherji S. Diffusion magnetic resonance imaging in the head and neck. Magn Reson Imaging Clin North Am 2011;19:55-67

15. Abdel Razek A. Diffusion-weighted magnetic resonance imaging of head and neck. J Comput Assist Tomogr 2010;34:808-15

16. Abdel Razek A, Elkhamary S, Mousa A. Differentiation between benign and malignant orbital tumours at 3-Tesla diffusion MR-imaging. Neuroradiology 2011;53:517-22

17. Sepahdari A, Aakalu V, Setabutr P, et al. Indeterminate orbital masses: restricted diffusion at MR imaging with echo-planar diffusion-weighted imaging predicts malignancy. Radiology 2010;256:554-64

18. Lope LA, Hutcheson KA, Khademian ZP. Magnetic resonance imaging in the analysis of pediatric orbital tumors: utility of diffusion-weighted imaging. $J$ AAPOS 2010;14:257-62

19. Politi L, Forghani R, Godi C, et al. Ocular adnexal lymphoma: diffusionweighted MR imaging for differential diagnosis and therapeutic monitoring. Radiology 2010;250:565-74

20. Rumboldt Z, Moses C, Wieczerzynski U, et al. Diffusion-weighted imaging, apparent diffusion coefficients, and fluid-attenuated inversion recovery MR imaging in endophthalmitis. AJNR Am J Neuroradiol 2005;26:1869-72

21. Al-Shafai LS, Mikulis DJ. Diffusion MR imaging in a case of acute ischemic optic neuropathy. AJNR Am J Neuroradiology 2006;27:255-57

22. Chen JS, Mukherjee P, Dillon W, et al. Restricted diffusion in bilateral optic nerves and retinas as an indicator of venous ischemia caused by cavernous sinus thrombophlebitis. AJNR Am J Neuroradiol 2006;27:1815-16

23. Jeh S, Kim S, Kim H, et al. Correlation of the apparent diffusion coefficient value and dynamic magnetic resonance imaging findings with prognostic factors in invasive ductal carcinoma. J Magn Reson Imaging 2011;33:102-09

24. Abdel Razek A, Gaballa G, Denewer A, et al. Invasive ductal carcinoma: correlation of apparent diffusion coefficient value with pathological prognostic factors. NMR Biomedicine 2010;23:619-23

25. Abdel Razek A, Fathy A, Abdel Gawad T. Correlation of apparent diffusion coefficient value with prognostic parameters of lung cancer. J Comput Assist Tomogr 2011;35:248-52

26. Edge SE, Byrd DR, Carducci MA, et al, eds. American Joint Committee on Cancer (AJCC) Staging Manual, 7th ed. New York, Springer-Verlag; 2009

27. Madhavan J, Ganesh A, Roy J, et al. The relationship between tumor cell differentiation and age at diagnosis in retinoblastoma. J Pediatr Ophthalmol Strabismus 2008;45:22-25

28. Eagle R. High-risk features and tumor differentiation in retinoblastoma: a retrospective histopathologic study. Arch Pathol Lab Med 2009;133:1203-09

29. Wippold F, Perry A. Neuropathology for the neuroradiologist: rosettes and pseudorosettes. AJNR Am J Neuroradiol 2006;27:488-92

30. James S, Halliday W, Branson H. Best cases from the AFIP. Trilateral retinoblastoma. RadioGraphics 2010;30:833-37

31. Provenzale J, Gururangan S, Klintworth G. Trilateral retinoblastoma: clinical and radiologic progression. AJR Am J Roentgenol 2004;83:505-11

32. Cuenca D, Giron F, Castro D, et al. Microscopic scleral invasion in retinoblastoma: clinicopathological features and outcome. Arch Ophthalmol 2009;127:1006-10

33. Wilson MW, Qaddoumi I, Billups C, et al. A clinicopathological correlation of 67 eyes primarily enucleated for advanced intraocular retinoblastoma. $\mathrm{Br} \mathrm{J}$ Ophthalmol 2011;95:553-58

34. Wilson M, Rodriguez-Galindo C, Billups C, et al. Lack of correlation between the histologic and magnetic resonance imaging results of optic nerve involvement in eyes primarily enucleated for retinoblastoma. Ophthalmology 2009; 116:1558-63 